\title{
A Fuzzy Evaluation Approach for Scientific Research Projects with 2-Tuple Linguistic Preference Relations
}

\author{
Huarong Zhang', Xiangqian Feng', * \\ ${ }^{1}$ Institute of science and technology, Nanjing Normal University, Nanjing, 210023, China \\ ${ }^{2}$ School of Business, Nanjing Normal University, Nanjing, 210023, China \\ * Corresponding author: Xiangqian Feng (Email: xiangqianone@126.com)
}

\begin{abstract}
Science and technology project evaluation is an important means of science and technology management. Whether the evaluation of science and technology projects is fair or not directly affects the development of national economy and the allocation of science and technology resources. Due to the complexity of the project and the fuzziness of human thinking, the expert information is often difficult to quantify in the process of project evaluation. Generally, the better choice is to express it in qualitative language. In this paper, the 2-tuple linguistic preference relation is proposed to evaluate scientific research projects. The reasonableness of the concept of complete consistency for the 2-tuple linguistic preference relation is discussed. Priority of 2-tuple linguistic preference relation is set up based on the 2-tuple weighted geometric averaging operator. Finally, combined with Science and technology project evaluation problem, the effectiveness and feasibility of the method are verified.
\end{abstract}

Keywords: Science and technology project evaluation; Linguistic preference relation; 2-tuple linguistic.

\section{Introduction}

Science and technology project initiation and evaluation is an important part of science and technology project management. It is an important means and guarantee to promote the sustainable and healthy development of national science and technology, promote the optimal allocation of science and technology resources, and improve the level of science and technology management [1]. Project initiation review is one of the core links of science and technology project evaluation. It is generally carried out in accordance with the procedures of preliminary review form review, peer expert communication review, comprehensive treatment of writing comprehensive review opinions, and discipline review meeting review. Peer expert review and comprehensive treatment are the two most important links in the review process [2]. Some scholars have studied using the uncertainty method to describe and gather the review opinions of peer experts. Dey [3] proposed a decision support system, which analyses projects with respect to market, technicalities, and social and environmental impact in an integrated framework using analytic hierarchy process, a multipleattribute decision-making technique. Zhu [4] proposed a project evaluation method for non-formatted Chinese text evaluation information based on multi-granular linguistic labels.

In the actual decision-making process, due to the fuzziness and diversity of science and technology project evaluation information, each expert's personal preference, professional status and authority are not exactly the same and other factors, it is impossible for experts to express different projects with certain values when comparing them. It is usually expressed in fuzzy language evaluation. The 2-tuple linguistic model formalized by Herrera and Martínez in year 2000, has upgraded several linguistic processes for solving complex decision-making issues. Its successful application in different fields has impelled several researchers to work in its extension. Faizi and Nawaz [5] think that the intuitionistic 2tuple linguistic set accurately deals with the imprecise and unpredictable information in those decision-making problems where experts prefer the degree of membership and nonmembership values in the form of 2-tuple. Faizi and Saabun [6] proposed Linear Best-Worst Method (LBWM) and Euclidean Best-Worst method (EBWM) to achieve the best criteria priority vector for Multi-Criteria Group Decision Making (MCGDM) problems in the context of intuitionistic 2-tuple linguistic information. Using 2-tuple to express the evaluation information of decision-makers is more practical, and more appropriately expresses the cognitive uncertainty with clear connotation and unclear extension in the process of scientific research project evaluation, which is conducive to the evaluation of scientific and technological projects with fuzzy characteristics. Therefore, this paper applies 2-tuple to the evaluation of science and technology projects, describes the evaluation information of science and technology projects with 2-tuple linguist terms, and puts forward a scientific and effective evaluation method of science and technology projects.

The rest of paper is organized as follows. In Section 2, we review some concepts and operations related to 2-tuple linguistic terms. In Section 3, gives the definitions of consistency for 2-tuple linguistic preference relation and then proposes the approach to derive the priority weights from 2tuple linguistic preference relations. In Section 4, the proposed approach is demonstrated by using an illustrative example. Finally, conclusions of this paper are drawn in section 5 .

\section{Preliminary}

Let $S=\left\{s_{i} \mid i \in T=\{0,1, \mathrm{~B}, \tau\}\right\} \quad$ be a finite and totally ordered linguistic term set with odd cardinality where $s_{i}$ represents a possible value for a linguistic variable, and the following characteristics should be satisfied [7]:

(1) $\min _{i \in T}\left(s_{i}\right)=s_{0}, \max _{i \in T}\left(s_{i}\right)=s_{\tau}$

(2) $s_{k}=\operatorname{Neg}\left(s_{\tau-k}\right)$; 
(3) $s_{k} \leq s_{l} \Leftrightarrow \Delta^{-1}\left(s_{k}\right) \leq \Delta^{-1}\left(s_{l}\right)$, where $\Delta^{-1}\left(s_{i}\right)$ is the index $i$ of the linguistic term $s_{i}(i=k, l)$;

(4) $\max \left(s_{k}, s_{l}\right)=s_{k}$, if $s_{k} \geq s_{l}$ and $\min \left(s_{k}, s_{l}\right)=s_{k}$, if $s_{k} \leq s_{l}$.

And some operational laws of any two linguistic terms $s_{k}, s_{l} \in S$ are introduced as follows:

(1) $s_{k} \oplus s_{l}=s_{k+l}$;

(2) $s_{k} \ominus s_{l}=s_{k-l}$;

(3) $\lambda s_{k}=s_{\lambda k}$;

(4) $\left(s_{k}\right)^{\lambda}=s_{k^{\lambda}}$;

(5) $\lambda\left(s_{k}+s_{l}\right)=\lambda s_{k}+\lambda s_{l}$;

(6) $\left(\lambda_{1}+\lambda_{2}\right) s_{k}=\lambda_{1} s_{k}+\lambda_{2} s_{k}$

Definition 1 Let $S=\left\{s_{i} \mid i \in T=\{0,1, \mathrm{~B}, \tau\}\right\} \quad$ be $\quad$ a linguistic term set and $\alpha \in[-0.5,0.5]$ denoting the symbolic translation, $\left(s_{i}, \alpha_{i}\right)$ is called a 2 -tuple linguistic variable, and the following characteristics should be satisfied [8]:

(1) $\Delta:[0, \tau] \rightarrow S \times[-0.5,0.5), \Delta\left(\beta_{i}\right)=\left(s_{i}, \alpha_{i}\right)$;

(2) $\Delta^{-1} S \times[-0.5,0.5) \rightarrow[0, \tau]$,

$\Delta^{-1}\left(s_{i}, \alpha_{i}\right)=i+\alpha_{i}=\beta_{i} \in[0, \tau]$

(3) $\operatorname{neg}\left(s_{k}, \alpha_{k}\right)=\Delta\left(\tau-\Delta^{-1}\left(s_{k}, \alpha_{k}\right)\right)$;

(4) $\left(s_{k}, \alpha_{k}\right)<\left(s_{l}, \alpha_{l}\right)$ if $k<l$;

(5) if $k=l$, (1)if $\alpha_{k}=\alpha_{l}, \quad\left(s_{k}, \alpha_{k}\right)=\left(s_{l}, \alpha_{l}\right)$,

(2)if $\alpha_{k}<\alpha_{l}, \quad\left(s_{k}, \alpha_{k}\right)<\left(s_{l}, \alpha_{l}\right)$

(3)if $\alpha_{k}>\alpha_{l}, \quad\left(s_{k}, \alpha_{k}\right)>\left(s_{l}, \alpha_{l}\right)$.

where $i=\operatorname{round}(\beta)$ and $\alpha_{i}=\beta-k, \alpha_{i} \in[-0.5,0.5)$.

Note "round" is the usual round operator.

And some operational laws of any two 2-tuple linguistic terms $\left(s_{k}, \alpha_{k}\right),\left(s_{l}, \alpha_{l}\right)$ are introduced as follows:

(1) $\left(s_{k}, \alpha_{k}\right)+\left(s_{l}, \alpha_{l}\right)=\Delta\left(\min \left\{g, \Delta^{-1}\left(s_{k}, \alpha_{k}\right)+\Delta^{-1}\left(s_{l}, \alpha_{l}\right)\right\}\right)$

(2) $\left(s_{k}, \alpha_{k}\right)-\left(s_{l}, \alpha_{l}\right)=\Delta\left(\max \left\{0, \Delta^{-1}\left(s_{k}, \alpha_{k}\right)-\Delta^{-1}\left(s_{l}, \alpha_{l}\right)\right\}\right)$

(3) $\lambda\left(s_{k}, \alpha_{k}\right)=\Delta\left(\min \left\{g, \lambda \Delta^{-1}\left(s_{k}, \alpha_{k}\right)\right\}\right)$;

(4) $\left(s_{k}, \alpha_{k}\right)^{\lambda}=\Delta\left(\min \left\{g,\left(\Delta^{-1}\left(s_{k}, \alpha_{k}\right)\right)^{\lambda}\right\}\right)$.

Definition 2. For linguistic 2-tuples $\left(s_{k}, \alpha_{k}\right),\left(s_{l}, \alpha_{l}\right)$, the deviation measure between $\left(s_{k}, \alpha_{k}\right)$ and $\left(s_{l}, \alpha_{l}\right)$ is defined as follows

$$
d\left(\left(s_{k}, \alpha_{k}\right),\left(s_{l}, \alpha_{l}\right)\right)=\left|\Delta^{-1}\left(s_{k}, \alpha_{k}\right)-\Delta^{-1}\left(s_{l}, \alpha_{l}\right)\right|
$$

\section{Consistency Measures and Priority for 2-Tuple Linguistic Preference Relation}

Definition 3 [9]. Let $P=\left(p_{i j}\right)_{n \times n}$ be a matrix. If $p_{i j}$ is a 2tuple, and $p_{j i}=\operatorname{Neg}\left(p_{i j}\right)$, then $P$ is called a 2-tuple linguistic preference relation.

Definition 4 Let $P=\left(p_{i j}\right)_{n \times n}$ be a 2-tuple linguistic preference relation, then $P$ is called a consistent 2-tuple linguistic preference relation if there exists $p_{i k} \oplus p_{k j}=p_{i j}$ for $i, j, k \in N=\{1,2, \mathrm{~B}, n\}$.

Theorem $1 P=\left(p_{i j}\right)_{n \times n}$ is called a consistent 2-tuple linguistic preference relation if and only if $Q=\left(q_{i j}\right)_{n \times n}$ is a consistent fuzzy preference relation, where

$$
q_{i j}=\left[0.5+\frac{\Delta^{-1}\left(p_{i j}\right)}{\tau}\right], i, j=1,2, \mathrm{~B}, n .
$$

Proof: Let $P=\left(p_{i j}\right)_{n \times n}$ is called a consistent 2-tuple linguistic preference relation, from Definition $4 p_{i k} \oplus p_{k j}=p_{i j}$ for $i, j, k \in N=\{1,2, \mathrm{~B}, n\}$, so

$$
\Delta^{-1}\left(p_{i k}\right)+\Delta^{-1}\left(p_{k j}\right)=\Delta^{-1}\left(p_{i j}\right) .
$$

Then

$$
\begin{aligned}
q_{i k}+q_{k j} & =\left[0.5+\frac{\Delta^{-1}\left(p_{i k}\right)}{\tau}\right]+\left[0.5+\frac{\Delta^{-1}\left(p_{k j}\right)}{\tau}\right] \\
& =\left[0.5+\frac{\Delta^{-1}\left(p_{i j}\right)}{\tau}\right]+0.5=q_{i j}+0.5
\end{aligned}
$$

Conversely, let $Q=\left(q_{i j}\right)_{n \times n}$ be a consistent fuzzy preference relation, then

$$
\begin{aligned}
\Delta^{-1}\left(p_{i k}\right)+\Delta^{-1}\left(p_{k j}\right) & =\tau\left(q_{i k}-0.5\right)+\tau\left(q_{k j}-0.5\right) \\
& =\tau\left(q_{i j}-0.5\right)=\Delta^{-1}\left(p_{i j}\right)
\end{aligned}
$$

So, $p_{i k} \oplus p_{k j}=p_{i j}$, which completes the proof of Theorem.

Lemma 1 [10] Let $P=\left(p_{i j}\right)_{n \times n}$ be a 2-tuple linguistic preference relation and $\bar{P}=\left(\bar{p}_{i j}\right)_{n \times n}$, where

$$
\bar{p}_{i j}=\Delta\left(\frac{1}{n} \sum_{k=1}^{n}\left(\Delta^{-1}\left(p_{i k}\right)+\Delta^{-1}\left(p_{k j}\right)\right) ; i, j=1,2, \mathrm{~B}, n,\right.
$$

then $\bar{P}$ is a consistent 2-tuple linguistic preference relation.

Definition 5 Let $P=\left(p_{i j}\right)_{n \times n}$ be a 2-tuple linguistic preference relation, then we define the distance between $P$ and $\bar{P}=\left(\bar{p}_{i j}\right)_{n \times n}$ as follows:

$$
d(P, \bar{P})=\frac{2}{n(n-1)} \sum_{j=i+1}^{n} \sum_{i=1}^{n} d\left(p_{i j}, \overline{p_{i j}}\right)
$$

We

set

$C I(P)=\frac{1}{\tau}(d(P, \bar{P}))=\frac{2}{n(n-1) \tau} \sum_{j=i+1}^{n} \sum_{i=1}^{n}\left|\Delta^{-1}\left(p_{i j}\right)-\Delta^{-1}\left(\overline{p_{i j}}\right)\right|$ as the consistency index of the 2-tuple linguistic preference relation $P$.

The above consistency index reflects the deviation degree between the linguistic preference relation $P$ and the consistent linguistic preference relations. Obviously, the smaller the value of $C I(P)$, the more consistent the linguistic preference relation $P$. If $C I(P)=0$, then $P$ is a consistent linguistic preference relation. If $C I(P)<0.1$, we conclude that $P$ is of acceptable consistency; otherwise, we conclude that $P$ is of unacceptable consistency.

Definition 6 [11]. Let TWGA: $S^{n} \rightarrow S$, if

$$
\operatorname{TWGA}_{v}\left(\left(s_{1}, \alpha_{1}\right),\left(s_{2}, \alpha_{2}\right), \mathrm{B},\left(s_{n}, \alpha_{n}\right)\right)=\Delta\left(\prod_{i=1}^{n}\left(\Delta^{-1}\left(s_{i}, \alpha_{i}\right)\right)^{v_{i}}\right)
$$

where $v_{i} \in[0,1]$ and $\sum_{i=1}^{n} v_{i}=1$, then TWGA is called the 2-tuple weighted geometric averaging operator. 
Let $P=\left(p_{i j}\right)_{n \times n}$ be a 2-tuple linguistic preference relation, we can obtained the weight vector $w=\left(w_{1}, w_{2}, \mathrm{~B}, w_{n}\right)$ by the following formula

$$
w_{i}=\frac{\sqrt[n]{\Delta^{-1}\left(T W G A_{v}\left(p_{i 1}, p_{i 2}, \mathrm{~B}, p_{i n}\right)\right)}}{\sum_{i=1}^{n} \sqrt[n]{\Delta^{-1}\left(T W G A_{v}\left(p_{i 1}, p_{i 2}, \mathrm{~B}, p_{i n}\right)\right)}}
$$

where $v_{i}=1 / n, i \in\{1,2, \mathrm{~B}, n\}$.

\section{An Illustrative Example}

In this section, taking the fund project evaluation of a basic research funding department as an example, it is assumed that there are 3 experts participating in the project evaluation. They give the following 2-tuple linguistic preference relation and try to select one for approval and funding from the four alternatives $X=\left\{x_{1}, x_{2}, x_{3}, x_{4}\right\}$ by using the linguistic terms in the set

$$
\begin{gathered}
S=\left\{\begin{array}{l}
s_{0}=\text { extremely poor, } s_{1}=\text { very poor, } s_{2}=\text { poor }, \\
s_{3}=\text { slightly poor, } s_{4}=\text { fair, } s_{5}=\text { slightly good }, \\
s_{6}=\text { good, } s_{7}=\text { very good, } s_{8}=\text { extremly good }
\end{array}\right\} \\
P=\left(\begin{array}{cccc}
\left(s_{4}, 0\right) & \left(s_{4},-0.3\right) & \left(s_{5},-0.4\right) & \left(s_{3}, 0.1\right) \\
\left(s_{4}, 0.3\right) & \left(s_{4}, 0\right) & \left(s_{5}, 0,1\right) & \left(s_{4},-0.4\right) \\
\left(s_{3}, 0.4\right) & \left(s_{3},-0.1\right) & \left(s_{4}, 0\right) & \left(s_{2}, 0.4\right) \\
\left(s_{5},-0.1\right) & \left(s_{4}, 0.4\right) & \left(s_{6},-0.4\right) & \left(s_{4}, 0\right)
\end{array}\right)
\end{gathered}
$$

Step 1 Consistency test for 2-tuple preference relationship. $\bar{P}=$

$$
\begin{aligned}
& \left(\begin{array}{cccc}
\left(s_{4}, 0\right) & \left(s_{4},-0.2\right) & \left(s_{4}, 0.3375\right) & \left(s_{4},-0.4375\right) \\
\left(s_{4}, 0.2\right) & \left(s_{4}, 0\right) & \left(s_{5},-0,4625\right) & \left(s_{4},-0.2375\right) \\
\left(s_{4},-0.3375\right) & \left(s_{3}, 0,4625\right) & \left(s_{4}, 0\right) & \left(s_{3}, 0.225\right) \\
\left(s_{4}, 0.4375\right) & \left(s_{4}, 0.2375\right) & \left(s_{5},-0.225\right) & \left(s_{4}, 0\right)
\end{array}\right) \\
& C I(P)=0.0495<0.1 \text {, we conclude that } P \text { is of }
\end{aligned}
$$
acceptable consistency.

Step 2 Priority for 2-tuple linguistic preference relation by the formula (4)

$$
w=(0.2407,0.2662,0.1970,0.2961)
$$

which means the ranking of alternatives is $x_{4}\left[x_{2}\left[x_{1}\left[x_{3}\right.\right.\right.$.

\section{Conclusion}

2-tuple linguistic is an effective tool to express humans' fuzzy information when providing linguistic evaluations, and have wide applications in MCDM. In this paper, we study the 2-tuple linguistic preference relation, which aim to evaluate scientific research projects. A simple yet pragmatic approach for judging whether or not a 2-tuple linguistic preference relation is consistency. In case of acceptable consistent 2tuple linguistic preference relation, the approach of deriving priority vector is put forward. The numerical results show that the proposed evaluation approach provides us with a useful way for scientific research projects.

\section{Acknowledgment}

The author is most grateful to the referees and the editors for their constructive suggestions. The work was partly supported by University Social Sciences Project of Jiangsu Province (No. 2016SJD630014).

\section{References}

[1] Jin M . Foreign Present Condition of the science and technology project evaluation and development trend research. International Journal of Business and Management, 2009, 3(8): 124-129.

[2] Wang X, Wu Q. Evaluation of scientific research fund projects based on fuzzy language. Scientific and technological progress and countermeasures, 2007, 24(9): 61-63.

[3] Dey P K . Integrated project evaluation and selection using multiple-attribute decision-making technique. International Journal of Production Economics, 2006, 103(1):90-103.

[4] Zhu J J, Wang H H, Ye C, et al. Project evaluation method using non-formatted text information based on multi-granular linguistic labels[J]. Information Fusion, 2015, 24:93-107.

[5] Faizi S, Nawaz S, Ur-Rehman A. Intuitionistic 2-tuple linguistic aggregation information based on Einstein operations and their applications in group decision making. Artificial Intelligence Review, 2020, 53(1): 4625-4650.

[6] Faizi S, Saabun W, Nawaz S, et al. Best-Worst method and Hamacher aggregation operations for intuitionistic 2-tuple linguistic sets. Expert Systems with Applications, 2021:115088.

[7] Rodríguez RM, Martínez L, Herrera F. Hesitant fuzzy linguistic term sets for decision making. IEEE Trans Fuzzy Syst, 2012,20(1):109-119.

[8] Herrera F, Herrera Viedma E, Martínez L. A fusion approach for managing multi-granularity linguistic term sets in decision making. Fuzzy sets and systems, 2000, 114( 1) : 43-58.

[9] Dong YC, Hong WC, Xu YF, Yu S. Selecting the individual numerical scale and prioritization method in the analytic hierarchy process: A 2-tuple fuzzy linguistic approach. IEEE Trans Fuzzy Syst, 2011, 19:13-26.

[10] Dong Y, Xu Y, Li H . On consistency measures of linguistic preference relations. European Journal of Operational Research, 2008, 189(2):430-444.

[11] Xu Y, Huang L. An Approach to Group Decision Making Problems Based on 2-Tuple Linguistic Aggregation Operators. International Colloquium on Computing, Communication, Control, \& Management. IEEE, 2008. 